\title{
CORONAL LOOPS AND ACTIVE REGION STRUCTURE
}

\author{
D. F. WEB B \\ American Science and Engineering, Inc., Cambridge, Mass. 02139, U.S.A. \\ and \\ H. ZIRIN \\ California Institute of Technology, Pasadena, Calif. 91125, U.S.A.
}

(Received 30 January; in revised form 27 May, 1980)

\begin{abstract}
We intercompared synoptic $\mathrm{H} \alpha, \mathrm{Ca} \mathrm{K}$, magnetograph and Skylab soft X-ray and EUV data for the purpose of identifying the basic coronal magnetic structure of loops in a 'typical' active region and studying its evolution. We focussed on a complex of activity in July 1973, especially McMath 12417. Our principal results are: (1) Most of the brightest loops connected the bright $f$ plage to either the sunspot penumbra or to $p$ satellite spots; no non-flaring X-ray loops end in umbrae; (2) short, bright loops had one or both ends in regions of emergent flux, strong fields or high field gradients; (3) stable, strongly sheared loop arcades formed over filaments; (4) EFRs were always associated with compact X-ray arcades; and (5) loops connecting to other active regions had their bases in outlying plage of weak field strength in McM 417 where $\mathrm{H} \alpha$ fibrils marked the direction of the loops. We conclude that a typical loop brightens in response to magnetic field activity at its feet, which heats the plasma. This suggests that the loop acts as a trap for gas convected from its base.
\end{abstract}

\section{Introduction}

\subsection{OBJectives}

Synoptic observations of the coronal component of active regions (ARs) are best obtained from above the Earth's atmosphere. Such observations from rockets and satellites have been made only in the last decade or so. Soft X-ray observations have played a significant role in studies of the coronal structure of ARs, because the corona can be viewed in high contrast against the solar disk. The earliest X-ray rocket imagery with adequate spatial resolution showed that the brightest AR emission occurred in a compact core structure which bridged the neutral line separating the dominant polarities (Krieger et al., 1971). Later observations resolved these structures as loops down to the limits of resolution. Van Spreybroeck et al. (1970) noted that ARs tended to group together in complexes of activity which were interconnected by long flux tubes, even across the equator. Thus, the magnetic flux emerging in a single AR was not entirely confined to that region, but was often shared with adjacent ARs or even the quiet network. Evolutionary studies have shown that the non-flaring X-ray emission from ARs could vary on time scales of hours and days and with amplitudes of a factor of 10 (Krieger et al., 1972), and that changes in loop form and brightness could be attributed either to changes in the temperature and density of existing flux tubes (Krieger et al., 1976), or to changes in the photospheric magnetic field (Howard and Švestka, 1977). 
Morphological studies intercomparing X-ray and EUV images of specific AR structures with $\mathrm{H} \alpha, \mathrm{CaK}$ and the magnetic field have been reported by Van Speybroeck et al. (1970), Krieger et al. (1971), Tousey et al. (1973), Sheeley et al. (1975), Neupert et al. (1975), and Smith et al. (1976). In this paper we extend these previous results by combining synoptic, high spatial resolution soft $\mathrm{X}$-ray, optical and magnetograph data to study the coronal loops and footpoints of one AR.

The objectives of this study were to examine the questions: What is the basic magnetic structure of a 'typical' active region? How do the loops which trace this structure in the corona evolve? What physical processes determine each phase of this evolution? Our approach was to assemble and analyze simultaneous data at different wavelengths on one well-observed AR complex during the Skylab period. We used this data set: (1) to identify individual loops or loop bundles and their footpoints with respect to the magnetic field, chromosphere and transition zone; (2) to study the evolution of these structures; (3) to classify AR loops in terms of their structure and evolution; (4) to try to understand the mechanism(s) of loop brightening; and finally (5) to study inter-region loop connections.

We concentrated on the structure of McMath region 12417, which reached CMP on July 5,1973 . This region was chosen because of high quality, comprehensive $\mathrm{H} \alpha$, $\mathrm{H} \alpha-0.7 \AA, \mathrm{Ca} \mathrm{K}$, and videomagnetograph (VMG) coverage combined with the availability of Skylab soft X-ray and EUV synoptic data. This classic active region complex has been discussed by Zirin (1974), Roy and Michalitsanos (1974), and Howard and Švestka (1977).

McM 417 had a relatively simple bipolar structure and covered a large area, making identification of structures easier. It was typical of the most common type of sunspot group, the $\alpha p$ group, with a dominant $p$ spot and a large, bright following plage separated by large fibrils and filaments. The region was part of an isolated complex of activity with long loops interconnecting a newly emerged region, $\mathrm{McM}$ 427, and a small, complex region, McM 414.

Next we review the evolution of the region. In Section 2 we describe the data base and our method of comparison. In Section 3 we present the results of the study, providing key examples of each result, and in Section 4 we summarize and discuss the results.

\subsection{EVOlution OF THE REGION}

McMath region 12417 was born in its previous rotation as McM 12375 on June 11, 1973. It had formed a complex bipolar region by west limb passage on June 14 and returned as McM 12417 on June 29 . By then it was a fairly simple $\alpha p$ group with a large dominant $p$ spot and two regions of extensive $f$ plage. A synoptic view of the region in X-rays and $\mathrm{H} \alpha$ is shown in Figure 1 for 4 days around CMP. One region which we call the ' $S f$ ' plage was directly south of and close to the spot. The other larger $f$ plage directly east, called the ' $E f$ ' plage, was separated from the $p$ spot and $p$ polarity to the north by a complex area that later formed a filament. Figure 2 presents three video-magnetograms on July 4, 6, and 8 which illustrate the evolution of the 


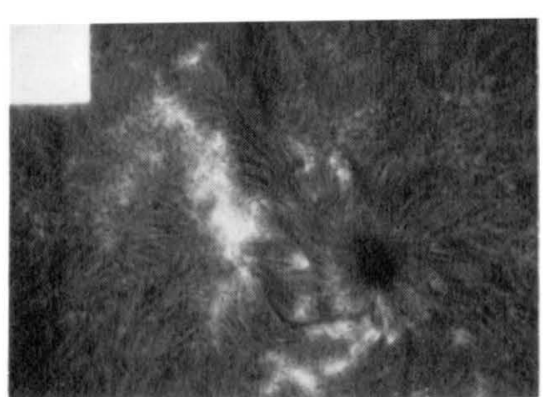

4, 2200 UT

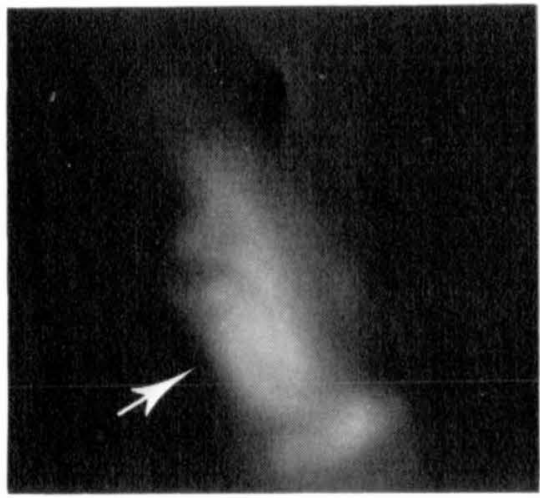

4, 2157 UT

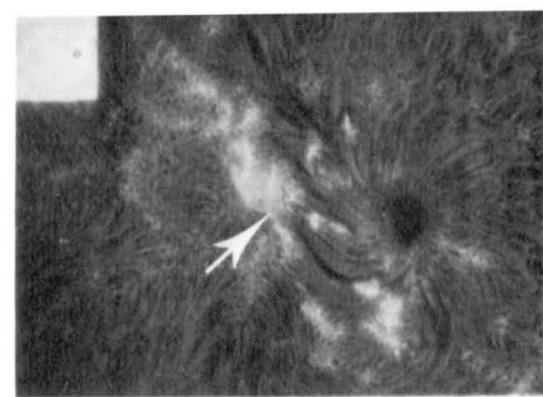

5, 2103 UT

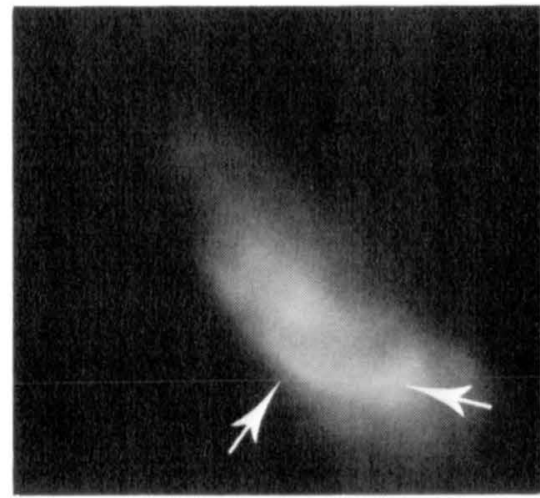

5, 2108 UT

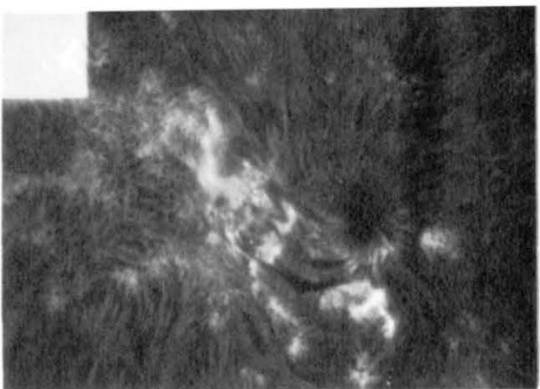

6, 2026 UT

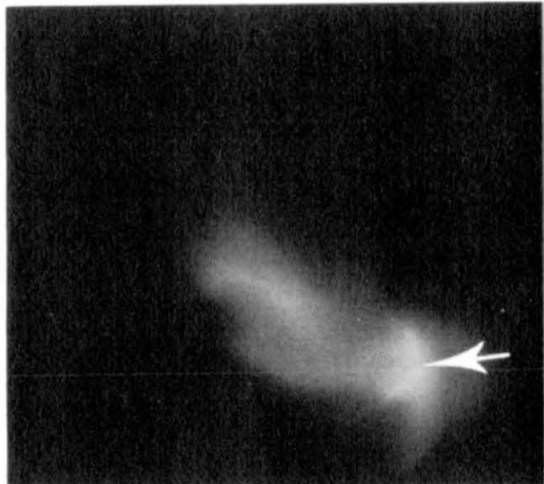

6, 2024 UT

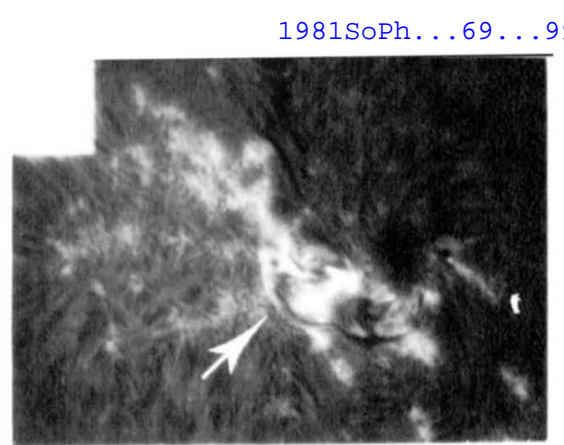

7, 1952 UT

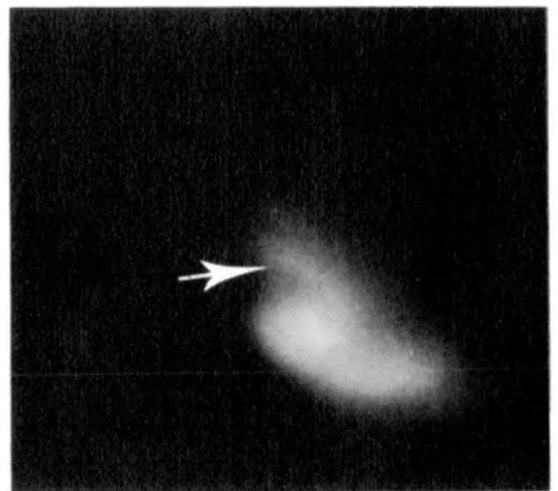

7, 1948 UT
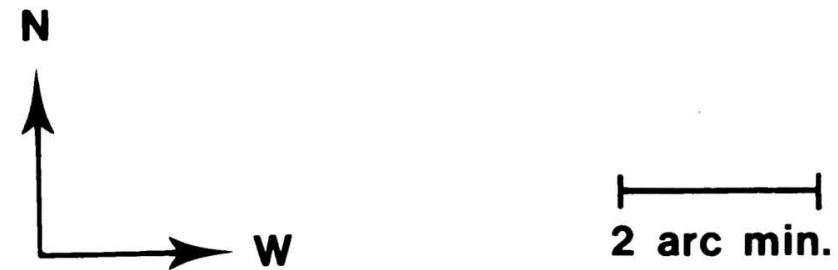

Fig. 1. Daily synoptic views of McMath 12417 on July 4-7, 1973, in $\mathrm{H} \alpha$ (top) and soft X-rays (bottom). The daily images are coaligned; the top of -the X-ray box is expanded to show the coronal arcade loops over the northern filament. Solar north is approximately up but the roll varies from day to day with the H $\alpha$ viewing area. The $\mathrm{H} \alpha$ filtergrams are from BBSO and are line center with a passband of $\frac{1}{4} \AA$. The X-ray images are $16 \mathrm{~s}$ exposures through filter 1 , a beryllium filter with a passband of $2-17 \AA$. 


\section{JULY 1973}

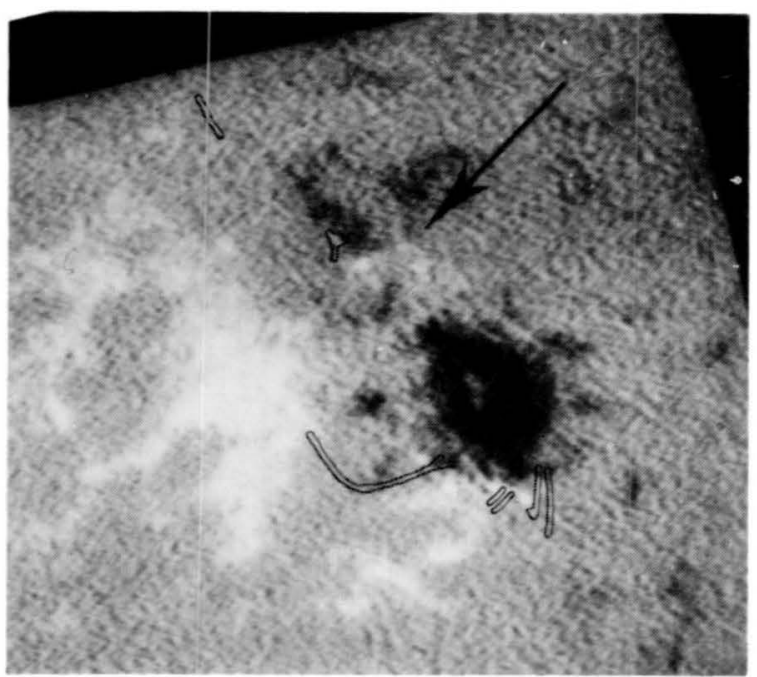

\section{4, 2213 UT}
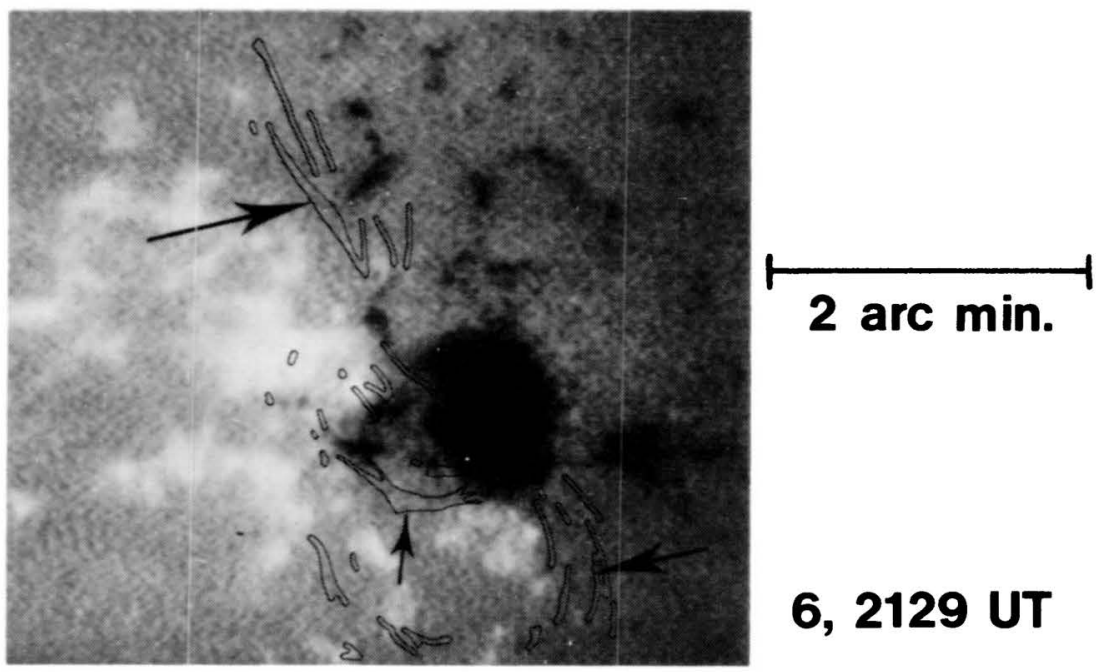

\section{6, 2129 UT}
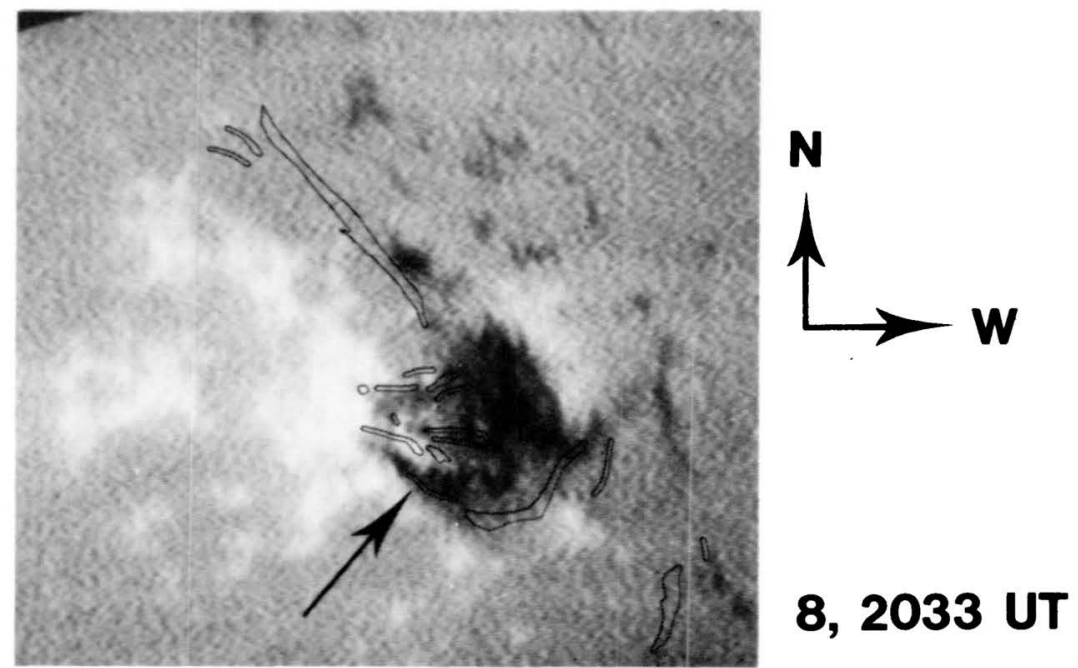

Fig. 2. Video-magnetograms of McMath 12417 in the $\mathrm{Fe}_{\mathrm{I}} 5324 \AA$ line. These illustrate the general magnetic evolution of the region. Black features correspond to $(-)$ polarity fields and white to $(+)$ fields. The filaments have been traced onto the magnetograms from simultaneous, coaligned $\mathrm{H} \alpha$ images (Table I). Note the single, large preceding spot (the magnetogram is saturated in this area) and the large area of following plage. 
photospheric magnetic field. Overlaid on each image are the $\mathrm{H} \alpha$ filaments which trace the polarity reversal lines through the region. Three large neutral lines divided the region and developed filaments along them (arrows: July 6). The large northern filament developed on this rotation and erupted on the next rotation in the well-known July 29 flare (Moore et al., 1980). The central east-west filament bridged the two zones of $f$ polarity to the east of the spot. The southern filament channel was sparsely populated by filament fragments in an area of weak field. On July 3-5 an area of moving $f$ polarity (arrow: July 4) was observed to move north from the vicinity of the spot, producing small flares as it interacted with neighboring $p$ polarity (Roy and Michalitsanos, 1974). However, the general magnetic configuration of the AR changed little until July 8 .

On July 7 an EFR appeared in the $f$ plage (H $\alpha$ arrow, Figure 1). On July 8 and 9 dynamic growth from this eruption transformed the region as an entire bipolar sunspot grew in the middle of McM 417 (Figure 2, arrow July 8). The rapidly moving new $p$ spot pushed aside old field, producing several sizeable flares at the leading edge, moved westward, then turned south as it encountered the old spot. When McM 417 rotated off the disk, it had two $p$ spots of roughly equal size.

McM 414, south of McM 416, was typical of many small, complex active regions that are difficult to classify. From east limb appearance on June 30 until July 3, the region exhibited two dipoles marching across the Sun. On July 4 strong flux emergence occurred in the following dipole, resulting in a triple neutral line structure as it rotated off the disk. McM 427 erupted onto the disk in a vacant area at about 16:00 UT on July 5. It rapidly evolved with flaring into a bipolar spot group, and almost as rapidly diminished.

\section{The Observations}

We compared simultaneous or near-simultaneous data using the Skylab American Science and Engineering soft X-ray (S-054) and Harvard College Observatory EUV (S-055) instruments, and ground-based optical data from the California Institute of Technology telescopes at Big Bear Solar Observatory (BBSO) in California and in Tel Aviv, Israel. Detailed comparisons were made for the active region complex from July 2-9, 1973, or for 3-4 days before and after CMP. Data near the solar limb was not used for detailed study because of foreshortening and the obscuration of coronal structures along the line of sight.

Table I presents the times of the images used for detailed examination as well as the total coverage in $\mathrm{H} \alpha$ and with the VMG. The $\mathrm{H} \alpha, \mathrm{H} \alpha$ blue wing and $\mathrm{Ca} \mathrm{K}$ data at BBSO were usually coincident. Results using the EUV data are described in Section 3.6 .

The X-ray data consisted of two-filter sequences of exposures taken every $12 \mathrm{hr}$, an observational mode used when Skylab was unmanned. The AS\&E experiment is described in detail in Vaiana et al. (1977). The images were recorded on film, had spatial resolution of a few arc sec, were disk-centered and viewed the full Sun. For 
TABLE I

Times of images used for the study (UT)

\begin{tabular}{|c|c|c|c|c|c|c|}
\hline Date (1973) & $\mathrm{X}-\mathrm{ray}^{\mathrm{a}}$ & $\mathrm{H} \alpha$ & VMG & $\mathrm{S}-055$ & $\mathrm{H} \alpha$ coverage & VMG coverage \\
\hline \multirow[t]{2}{*}{ July 2} & $13: 27$ & $13: 23$ & - & $13: 47$ & $00: 00-02: 35$ & \\
\hline & $21: 43$ & & & & $07: 37-24: 00$ & $16: 44-24: 00$ \\
\hline \multirow[t]{2}{*}{ July 3} & $11: 09^{b}$ & & & & $00: 00-02: 15$ & $00: 00-01: 13$ \\
\hline & $21: 07$ & $21: 01$ & $20: 53$ & & $07: 07-24: 00$ & $13: 47-24: 00$ \\
\hline \multirow[t]{2}{*}{ July 4} & $13: 39$ & $13: 44$ & & $13: 56$ & $00: 00-02: 15$ & $00: 00-02: 17$ \\
\hline & $21: 58$ & $22: 00$ & $22: 13$ & & $13: 31-24: 00$ & $14: 01-24: 00$ \\
\hline \multirow[t]{2}{*}{ July 5} & $13: 10$ & $11: 08$ & $15: 56$ & & $00: 00-02: 00$ & $00: 00-01: 57$ \\
\hline & $21: 09$ & $21: 03$ & $21: 16$ & & $08: 08-24: 00$ & $15: 53-24: 00$ \\
\hline \multirow[t]{3}{*}{ July 6} & $13: 52$ & $11: 50$ & & $14: 08$ & $00: 00-02: 20$ & $00: 00-00: 06$ \\
\hline & & $15: 10$ & & & & \\
\hline & $20: 25$ & $20: 26$ & $21: 29$ & & $07: 04-24: 00$ & $16: 12-24: 00$ \\
\hline \multirow[t]{3}{*}{ July 7} & $13: 08$ & $14: 07$ & & & $00: 00-02: 15$ & $00: 00-00: 43$ \\
\hline & & $14: 43$ & & & & \\
\hline & $19: 49$ & $19: 52$ & $19: 39$ & $19: 40$ & $13: 14-24: 00$ & $16: 43-24: 00$ \\
\hline \multirow[t]{3}{*}{ July 8} & $14: 02$ & $13: 18$ & & & $00: 00-02: 23$ & \\
\hline & $20: 35$ & $20: 25-20: 45$ & $20: 33$ & & & \\
\hline & & & & & $08: 05-24: 00$ & $15: 10-24: 00$ \\
\hline \multirow{2}{*}{ July 9} & $13: 19$ & & & & $00: 00-02: 18$ & $00: 00-00: 47$ \\
\hline & $21: 28$ & $21: 10$ & & & $07: 41-24: 00$ & $13: 31-19: 56$ \\
\hline
\end{tabular}

a Time of the X-ray image obtained with filter $1(2-17 \AA)$ at the end of a $64 \mathrm{~s}$ exposure.

b Time of $16 \mathrm{~s}$, filter $3(2-32,44-54 \AA)$ exposure.

this study we used primarily filter 1 images $(2-17 \AA)$, which were optimal for observing the bright, compact structures within active regions.

We used two kinds of enlarged S-054 transparencies. The first kind were density images enlarged from the original film; the second kind were computer-enhanced images. X-ray telescopes inherently have a large amount of scatter which tends to obscure details in bright, complex sources like active regions. The enhanced images were produced with a fast-Fourier transform technique which filters low spatial frequencies from a scanned density image to yield an image of sharper definition. This technique is described in Kahler et al. (1981) where several examples are shown. Such an image is shown in Figure 5 of this paper. We found the enhanced images to be invaluable in locating individual loops and tracing their footpoints.

The combination of two dedicated observatories on opposite points of the Earth and excellent weather permitted almost continuous optical coverage of these solar regions from June 30 to July 10 (Table I). The Tel Aviv observations were in $\mathrm{H} \alpha$ only and covered about a quarter of the disk (using a $50 \mathrm{~mm}$ disk-diameter image). The BBSO data used a full disk patrol with a Fabry Perot $\mathrm{H} \alpha$ filter (resolution about 2 arc-sec) and two $25 \mathrm{~cm}$ refractors feeding 3 cameras. The latter was set for two channels of $\mathrm{H} \alpha$ (variously center line and wing, or opposite wings) and the $\mathrm{Ca}$ II $\mathrm{K}$ line. The spatial resolution of this system was typically $0.5 \mathrm{arc} \mathrm{sec}$, with a projected area of $4.0 \times 5.2 \mathrm{arc} \min$ and time resolution of usually $10 \mathrm{~s}$. One of the $25 \mathrm{~cm}$ telescopes also fed a videomagnetograph (VMG) with $2 \mathrm{arc} \mathrm{sec} \mathrm{resolution.} \mathrm{The} \mathrm{Tel}$ 
Aviv and BBSO full disk patrol observations contained all three regions of interest. The $25 \mathrm{~cm}$ data were mostly centered on McM 417 with occasional sequences on 414 and 427. Previous $\mathrm{H} \alpha$ and VMG observations of McM 417 have been published by Zirin (1974) and Roy and Michalitsanos (1974).

All of the primary data (Table I) were enlarged to a scale of a 16-inch $(40.6 \mathrm{~cm})$ solar disk diameter. Eighteen-cm scale images were also available. X-ray to $\mathrm{H} \alpha$ registration was achieved by using an intermediary visible.light S-054 image to align to the large sunspot and pores in McM 417. The largest source of alignment error was in rotation. On days when other spots or pores were present, we estimated the overall positional accuracy was $\sim 5 \mathrm{arc} \mathrm{sec}$. On other days it could have been $\geq 10 \mathrm{arcsec}$. The EUV data were aligned directly to the $\mathrm{H} \alpha$ images with an accuracy no better than the 5 arc sec EUV pixel size. These data were supplemented by cine versions of the $\mathrm{H} \alpha, \mathrm{Ca} \mathrm{K}$, and VMG data.

\section{Results}

We believe that the following conclusions describe the basic nature of the coronal loops and their footpoints. These results apply in particular to the brighter, more easily defined inner loops of the $A R$, and to the outer or interconnecting loops which were less obscured by the bright core of the AR.

\subsection{The BRIGHT, INTERNAL STRUCTURE}

Examination of the X-ray and $\mathrm{H} \alpha$ data of McM 417 revealed the following general facts: (1) the brightest chromospheric emission appeared over the $f$ portion of the $\mathrm{AR}$; (2) the brightest X-ray (coronal) emission was over $\mathrm{H} \alpha$ structures where the field direction could be interpreted as horizontal, e.g., the loops were essentially parallel to the $\mathrm{H} \alpha$ fibril structure; and (3) every brighter $\mathrm{H} \alpha$ area had particularly bright loops either rooted in it or crossing it.

The bright $\mathrm{X}$-ray emission over the $f$ plage probably consisted of families of loops ranging from small, unresolved structures to large bundles of loops. Typically these inner loops connected the bright $f$ plage to either the penumbra or to satellite $p$ polarity east of the main spot.

No loop emitting at coronal temperatures had a foot in the spot umbra. This observation applied generally to ARs with well-developed sunspots as viewed in $\mathrm{X}$-rays (Pallavicini et al., 1979). Of course, we expect many loops to emanate from the umbral region, but they are somehow cooled by the spot. In McM 417 the convergence of lines of force in the large spot umbra left little $p$ plage outside the umbra and a corresponding absence of emission in the $p$ field region*. In bipolar spot regions the emission is more symmetrical (e.g., Figure 6).

\footnotetext{
* Some flux in a typical AR is shared with other regions, as evidenced by interconnecting loops (Section 3.5). However, since in a large AR such as McM 417 these loops terminate in weak field patches, the fraction of flux shared is small.
} 
We cite several examples of the internal loop structure as observed near CMP (July 4-6). Late on July 4 three prominent X-ray arches appeared extending from $f$ plage to satellite $p$ spots in the area of $p$ polarity intrusion to the east of the spot (arrow, Figure 1). Two of these arches had their $f$ footpoints in bright X-ray knots. One of these knots and the third loop foot lay in areas of undistinguished plage. The other bright knot was cospatial with a tiny $f$ pore. Thus pores appeared at one or both footpoints of all these bright arches. These observations show that intermittent pores can support hot coronal loops, while large, stable spots do not. This class of pores is shortlived and is associated with continuous activity and bright plage.

An extensive flare on July 5 at $01: 20$ UT resulted in a simplification of the above region. The X-ray images on July 5 were dominated by long loop structures extending from the penumbra to $f$ plage. During July 6 three new pores developed, with two in the general area of the bright $f$ plage above (arrows, Figure $5 \mathrm{c}$ ). By the next day numerous small pores or spots of both polarities developed in this region, which became dominated by emerging flux on July 8. On July 7, 19:49 UT, the second brightest X-ray feature was a loop (arrow, Figure 1) again connecting this area of intruding $p$ flux with older $f$ plage. The brightening of this loop, not previously obvious, appeared to be associated with the activity in the $p$ footpoint region.

\subsection{INTENSE LOOPS ARISING FROM REGIONS OF HIGH FLUX CONCENTRATION}

Several persistent low-lying, bright loops connected $p$ polarity in the southern part of the penumbra to the bright $S f$ plage. One loop (arrow, Figure 1, July 5; D in Figure 3 ) was a prominent feature in X-rays from late on July 4 to midday on July 6 . We estimate that the loop was $3.5 \times 10^{4} \mathrm{~km}$ in length. At its penumbral footpoint was an area of persistent activity in the form of $\mathrm{H} \alpha$ brightenings. The southern $f$ footpoint was an area of strong field and high field gradients as evidenced by dark $f$ pores that became visible by late on July 6 .

This area of the $S f$ plage was active during the first days of McM 417's disc passage. The earliest data that was not seriously foreshortened was on July 2. From July 2 to mid-July 4, we observed a classic, small AFS (arch filament system) in this area. As we observed with other EFRs (Section 3.4), a very bright but tiny $\left(1.0 \times 10^{4} \mathrm{~km}\right.$ ) $\mathrm{X}$-ray loop crossed the AFS. Figure 4 shows the relationship of this loop to $\mathrm{H} \alpha$ on July 3 at about 21:00 UT. The loop was aligned and cospatial with field lines as traced by the arch filaments in $\mathrm{H} \alpha$. The loop was accurately aligned with $\mathrm{H} \alpha$ because both chromospheric footpoints were near flare brightness (Arrow 1). We used the $\mathrm{H} \alpha$ cine coverage of flaring points to help us in locating the footpoints of this and other loops. Another example of this technique is Figure $4 \mathrm{~b}$ which shows coincident flaring ribbons (arrows 2 and 3). These ribbons suggested a connecting loop or loops, which indeed we saw in the earlier X-ray image at 21:06 UT (arrow, Figure 4c). Figure 4d is an overlay sketch showing the relationship of these loops to their bases in the lower atmosphere. 


\section{JULY 1973, 2115 UT}
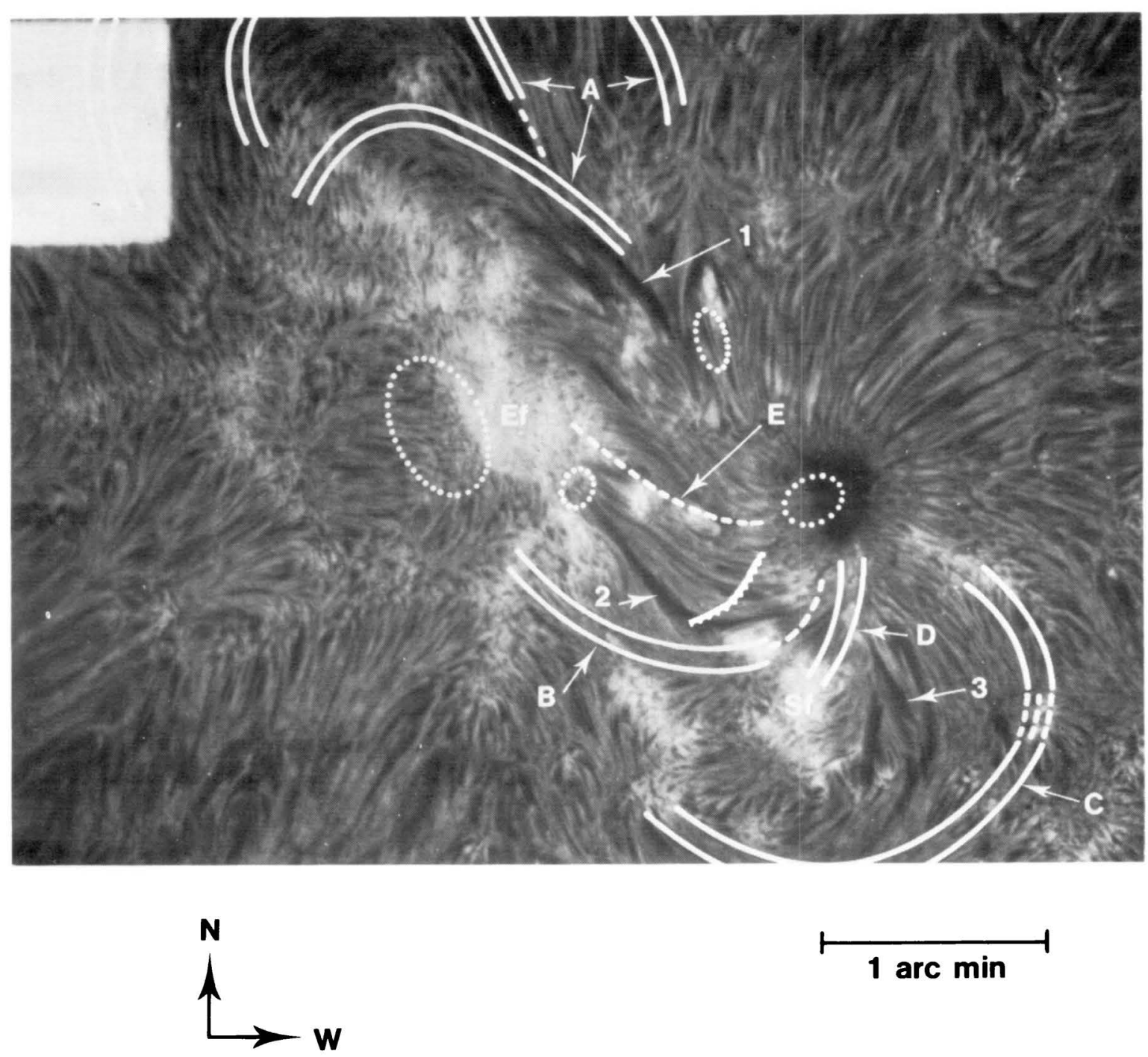

1 arc min

Fig. 3. The overall appearance of McMath 12417 at CMP on July 5. The overlay is a schematic representation of the brightest X-ray structures from an image at 21:09 UT. Solid lines are entire loop structures, dashed lines show possible loops or loop extensions, and the dotted ovals are amorphous patches of emission. Loop $\mathrm{E}$ and the emission near the umbra are probably associated with an umbral flare (Tang, 1978). The numbers designate the three filament channels; the letters show the persistent $\mathrm{X}$-ray loops associated with these areas. ' $E f$ ' and ' $S f$ ' designate the extensive and enduring following polarity plage east and south, respectively, of the dominant $p$ spot. Compare with the photospheric field of Figure 1.

The tiny X-ray loop in Figure 4 was not the same as Loop D (Figure 3) though it was in about the same location. It was shorter than Loop D, and, more important, the magnetic configuration was reversed. An area of $S f$ plage intruded into the edge of the penumbra on July 2 and 3 . The AFS connected this area of $f$ polarity with a $p$ spot to the south (Figure 4d). By late on July 4 this entire area had changed to an apparently more stable configuration whereby the lines of force ran $\mathrm{N}-\mathrm{S}$ from the penumbra to the $S f$ plage. Persistent Loop D is an indication of this more stable 


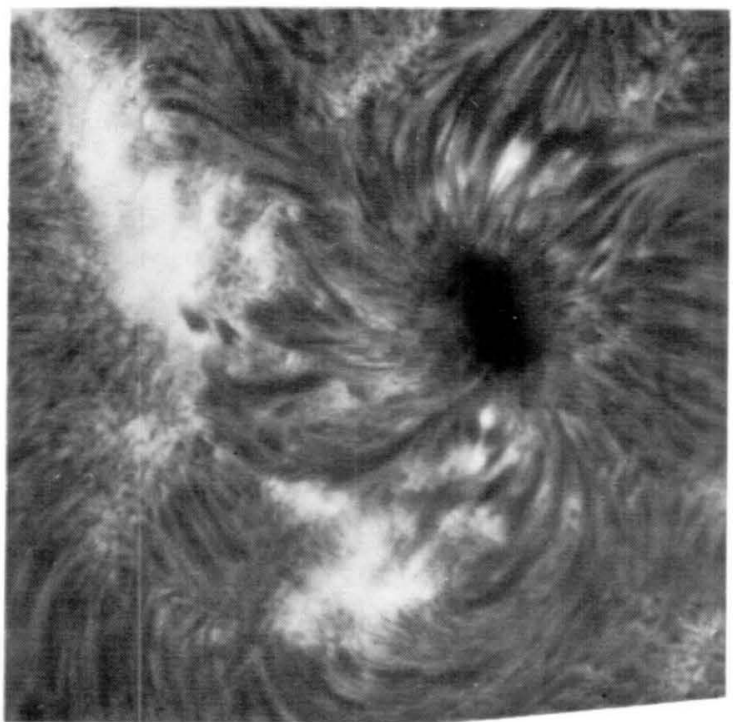

(a)

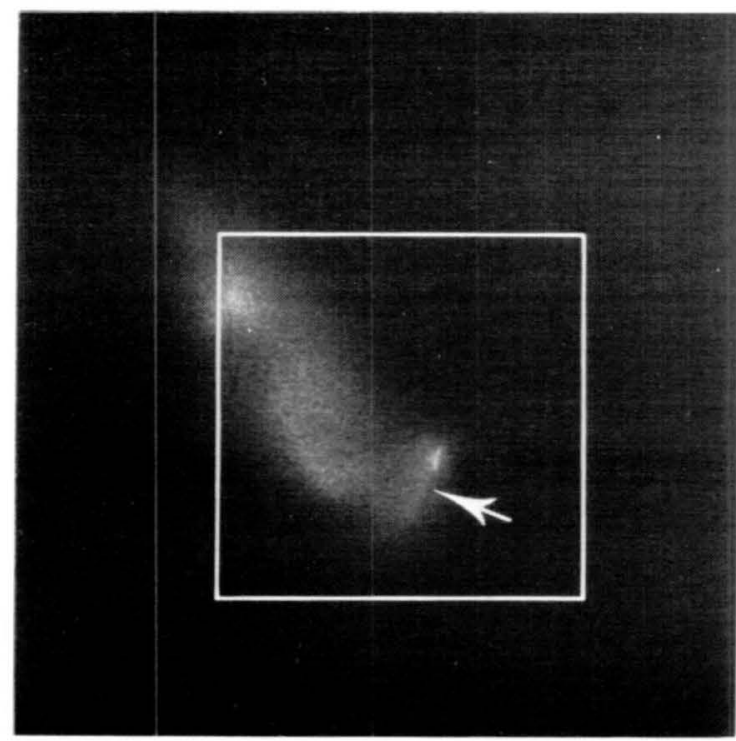

(c)
2114 UT

2106 UT

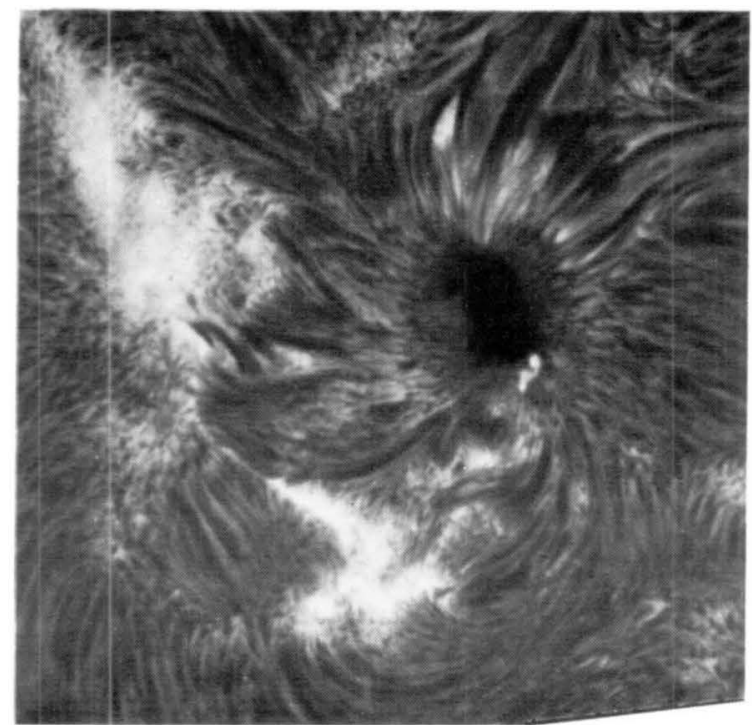

(b)

2227 UT

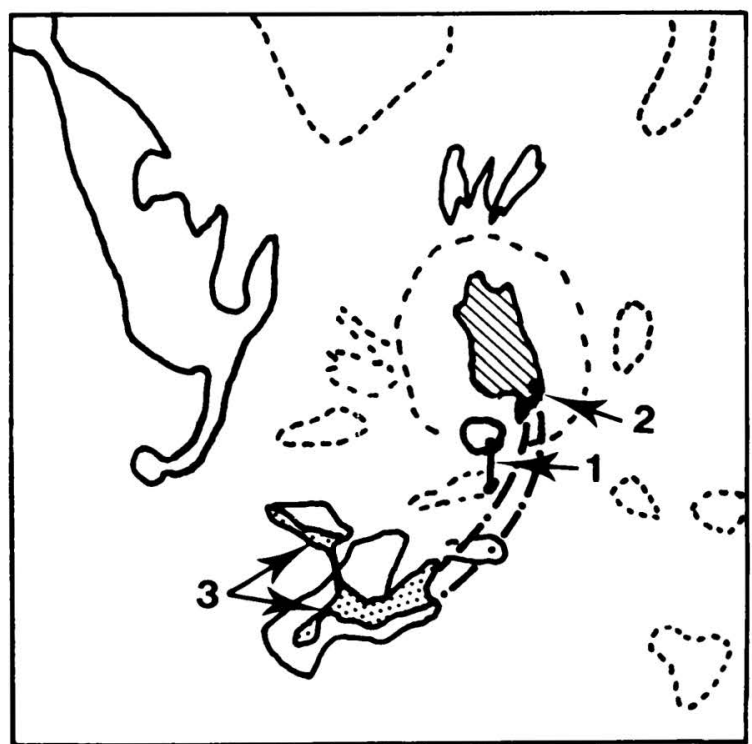

(d)

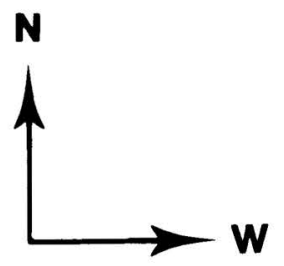

Fig. 4. McMath 12417 in H $\alpha$ from BBSO (top) and soft X-rays (c) late on July 3, 1973. Panels (a), (b), and (d) are enlarged $2 \times$ with respect to the $X$-ray image; the inset box in (c) represents the equivalent area of the other panels. (d) is a schematic drawing showing our interpretation of the magnetic configuration of the region. The dashed outlines and the sunspot are $p$ polarity and the solid outlines are $f$ polarity. Arrow 1 shows an AFS (a) whose feet were brightly emitting in $\mathrm{H} \alpha$; the tiny, bright X-ray loop in (c) was contemporary and cospatial with the AFS. Arrows 2 and 3 denote conjugate flaring $\mathrm{H} \alpha$ ribbons (b) which were probably joined by a pre-existing coronal loop seen 80 min earlier (arrow, panel (c)). 
configuration, and appeared late on July 4 after the existing field had accommodated the AFS.

Another example of a persistent loop connection across this stable magnetic configuration was a N-S loop that lay just west of Loop D. This loop was first clearly seen in X-rays on July 6, 20:25 UT (Figure 1), after Loop D had faded, and was brightest on July 7, 13:08 UT. The ends of this loop probably are illuminated by the two-ribbon flare illustrated in the $\mathrm{H} \alpha$ image on July 3 at $22: 27: 27$ UT in Figure 4b. The northern foot lay at or near an area of persistent activity on July 3 and 4 near the edge of the umbra. This activity was in the form of bright emission, flaring and surging. Another loop of this class connected the eastern part of the penumbra to an area of recurrent activity in the $E f$ plage ( $\mathrm{H} \alpha$ arrow, Figure 1, July 5).

\subsection{Filament ARCAdes}

Three persistent neutral lines dominated the region throughout this rotation. Figure 3 shows the overall appearance of the AR in $\mathrm{H} \alpha$ near CMP. Arrows 1, 2, and 3 delineate the three filaments or filament channels. The dominant and most stable filament channel was No. 1, which persisted during the entire rotation and was associated with stable X-ray arches with a high degree of shear. A stable coronal arcade appeared to overlie the northern filament during this period, although at any one time only certain arches emitted in X-rays. The coronal arch system was present at east limb passage and was well developed by late on July 4 , even though the filament reached maximum darkness days later.

The broadest and brightest arch appeared on July 6 at 13:52 UT (Figure 5d, Arrow A). One footpoint lay in the northern bright $f$ plage and the other in $p$ polarity in the midst of an active area of mixed polarity to the north of the spot (Figure 2). In this latter area was the moving rim of $f$ polarity field described by Roy and Michalitsanos (1974). On July 5 and 6 several of the brightened filament arches had their western feet in the area of activity produced by the rim. It is possible that this activity heated the arches and provided the loops with material, which then condensed as the filament.

The locus of $p$ footpoints of the arcade loops described a line running parallel to and closely adjacent to the neutral line, indicating a high degree of shear of the field over the filament. The arcade loops also appeared to have an inflection, implying one or more twists in them (Figure 5d). These observations suggest that sheared loops can exist in ARs in long-term stable configurations. In general the arcade appears more sheared as it approaches the strong fields of the spot, and the loops tend to be higher and more potential-like farther from the spot.

Filament No. 2 was a persistent magnetic feature which separated the bright $S f$ plage from satellite $p$ polarity following the large spot (Figures 1 and 2). This filament was visible in $\mathrm{H} \alpha$ on every day, even after the development of the EFR on July 8 . The most persistent, bright X-ray structure we observed was a bright arch (Figure 3, Arrow B) which was slightly longer than this filament and inclined to the neutral line at a small angle $\left(\sim 10^{\circ}\right)$. This arch was often the brightest X-ray feature in the AR 
(arrow, Figure 1, July 5). Its western footpoint was in the penumbra and its eastern foot in an area of bright $f$ plage where persistent activity occurred and $f$ pores developed.

Filament No. 3 was oriented N-S and separated the $S f$ plage from $p$ polarity spreading from the big spot. It was stable throughout this rotation and had arches of variable intensity overlying it (Figures 3 and 5), which appeared to be in planes highly

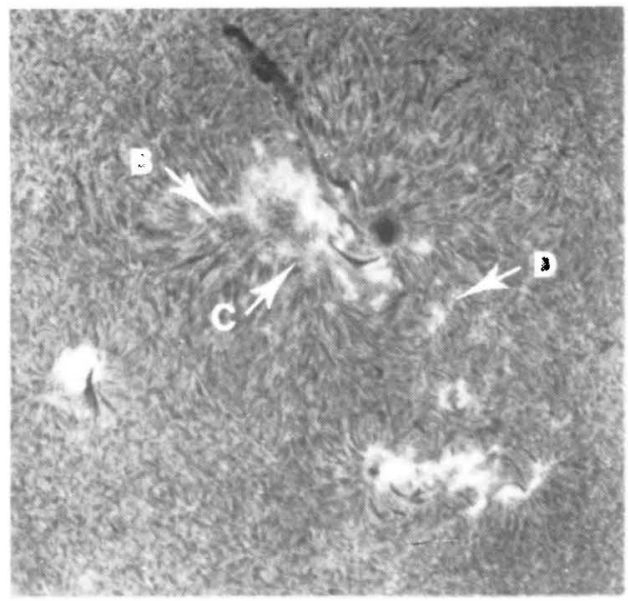

(a)

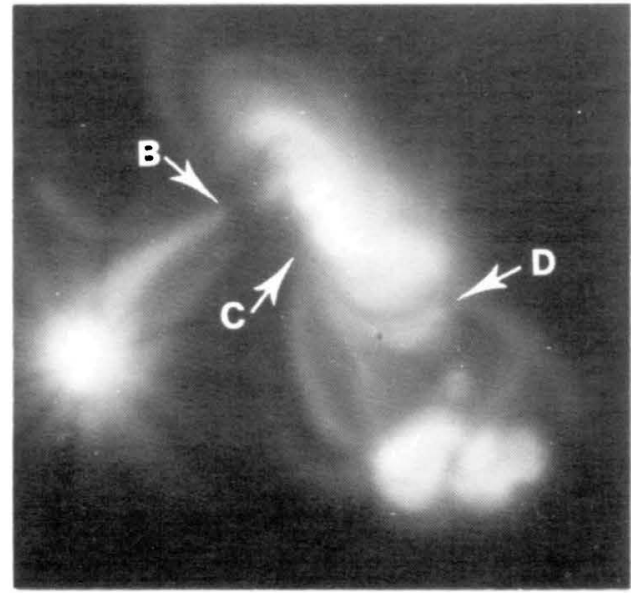

(b)
1151 UT

1357 UT
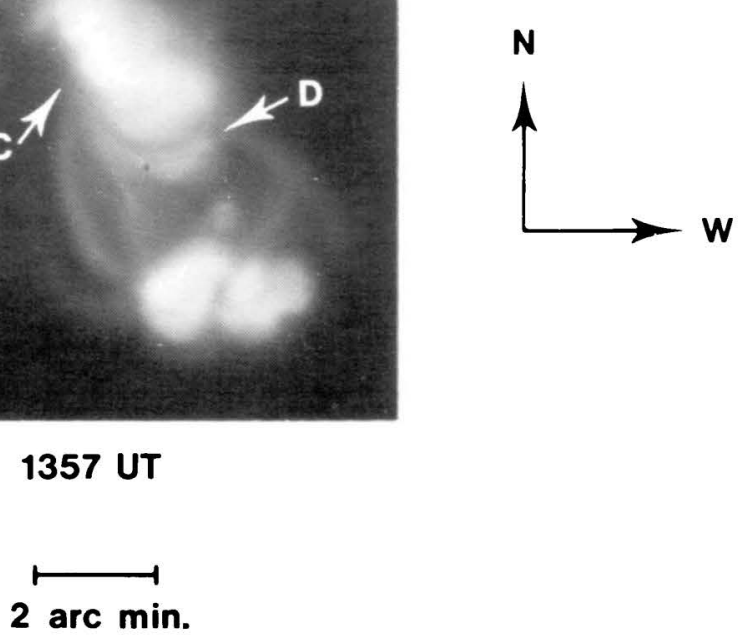

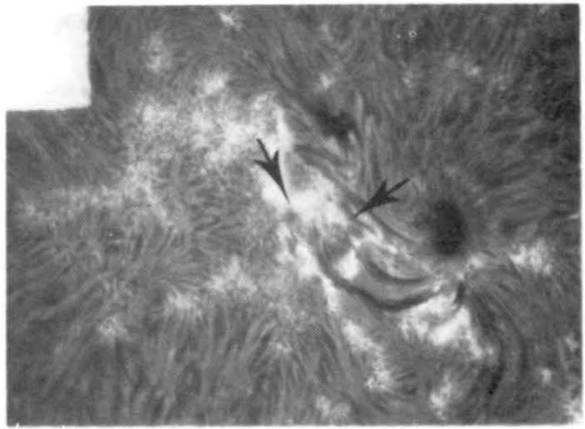

(c)

1654 UT

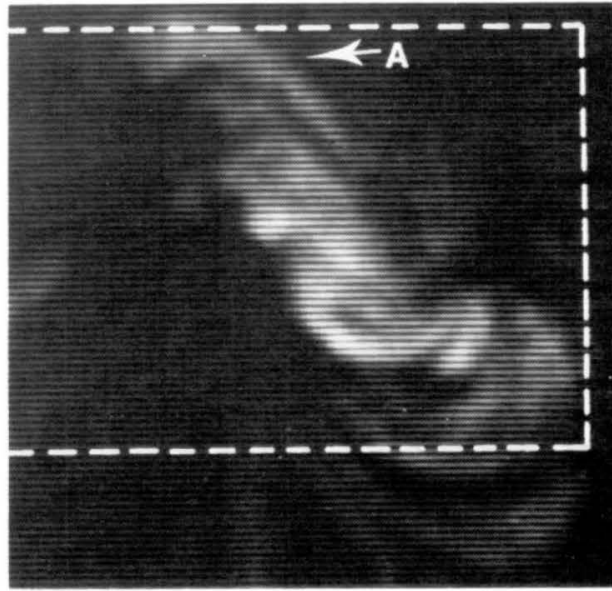

(d)

1352 UT

2 arc $\min$.

Fig. 5. The active region complex on July 6,1973 in $\mathrm{H} \alpha$ (top) and soft X-rays (bottom). The left side shows a large-area view of the three interconnected regions: McM 427, east of center, McM 417, north, McM 414, southwest. (a) is an $\mathrm{H} \alpha$ filtergram from Israel, at line center with a passband of $\frac{1}{2} \AA$. (b) is a $16 \mathrm{~s}$ exposure through Filter 3 (passband of 2-32, 44-54 $\AA$ ). Coaligned arrows $B, C$, and D point to the McM 417 terminations of the interconnecting loops. The right side is an enlarged view of McM 417. (c) is a full field BBSO $\mathrm{H} \alpha$ image; the arrows denote two new $f$ pores. (d) is a Filter 1 digital, enhanced version of the 417 region in (b). The X-ray box size has the same scale but is larger than the $\mathrm{H} \alpha$ box; the dashed outline marks the position of the $\mathrm{H} \alpha$ box on the X-ray field. Arrow A shows a bright arcade arch overlying filament No. 1 (Figure 2). 
inclined to the vertical. The feet of these loops were in areas of weaker magnetic field (Figure 5).

\subsection{DeVElopMent of EMERGing FLUX REgions}

Three large emerging flux regions (EFRs; Weart, 1970) appeared during this rotation: that which became McM 427, a sizeable EFR in the following part of McM 414 starting July 4 after $\sim 15: 00$ UT, and the large EFR that transformed McM 417.

The close correlation of compact X-ray structures with EFRs was noted by Krieger et al. (1976). Each of the EFRs we observed was bright in the first X-ray image available after the EFR became visible in $\mathrm{H} \alpha$. The EFR in McM 417 was continuously bright because of many flares and probable reconnections with the strong magnetic fields in which it emerged. The EFR in McM 414 was never very bright in $\mathrm{X}$-rays; there were always other brighter structures in the region.

In Figure 6 we show two sequences of the EFR in McM 414 with $\mathrm{H} \alpha$ and X-ray pairs. The early development is shown in Figure 6 at about 22:00 UT on July 4; Figure $6 \mathrm{~b}$ shows the next X-ray image on July 5 at 13:08 UT with an earlier Tel Aviv $\mathrm{H} \alpha$ image, chosen because of better seeing. The X-ray loops followed the oval pattern of the $\mathrm{H} \alpha$ arches and were brightest where the field could be interpreted as horizontal. The loops were cospatial with and aligned along the E-W direction of the dark fibrils. There was no correspondence, however, between the brightest X-ray loops and the dark fibrils. Unexpectedly, except during flares, the $\mathrm{H} \alpha$ plage emission and the coronal loop brightenings were not well correlated in time.

Note how the coronal loops converged on the sunspots. There is no suppression of coronal emission in loops going into a spot until a long-lived mature sunspot is formed. Coronal emission is not a function of penumbral formation; small mature spots without penumbra in McM 414 show no coronal emission over them. We conclude that as long as spots are emerging and growing, enough energy is generated to heat the coronal loops embedded in them, but once the stable spot forms, loop heating does not occur.

The growth of McM 427 indicates the way coronal heating above an EFR develops. A small, intense X-ray source appeared on the first available image $5 \mathrm{hr}$ after the EFR became visible in the $\mathrm{H} \alpha$ cine data on July 5, 16:00 UT. The initial coronal heating was coincident with EFR emergence; the coronal cloud expanded approximately with the $\mathrm{H} \alpha$ emission size of the region. However, an X-ray image on July 5, 13:16 UT, revealed a small knot at the subsequent location of the EFR, indicating that: (1) the EFR was more easily observable in the high contrast X-rays; (2) coronal heating actually preceded chromospheric brightening; or (3) the correspondence of the knot and the EFR was coincidental.

\subsection{INTERCONNECTING LOOPS}

The three regions were interconnected by a number of loops. Chase et al. (1976) described the properties of such X-ray loops, and Howard and Švestka (1977) discussed the interconnections in the 417 complex using $\mathrm{X}$-ray and magnetograph 


\section{JULY 1973}

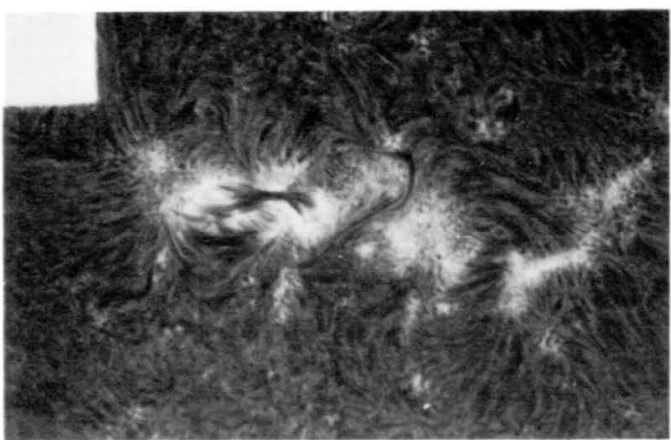

4, 2225 UT

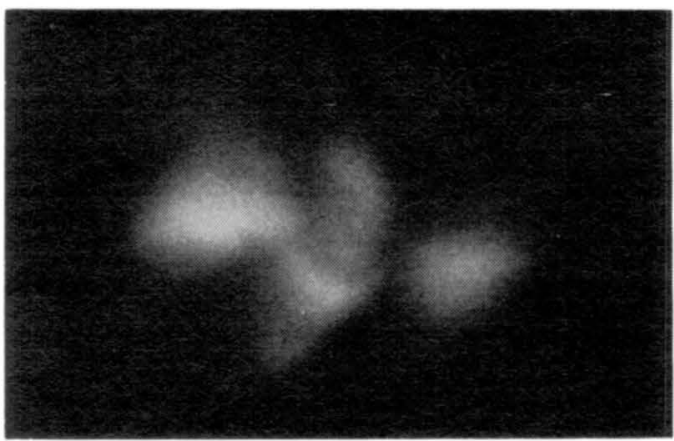

4, 2158 UT

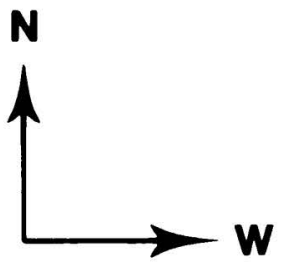

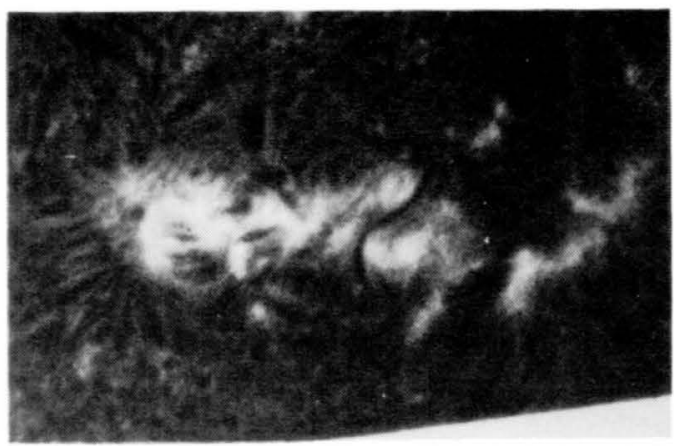

5, 1108 UT

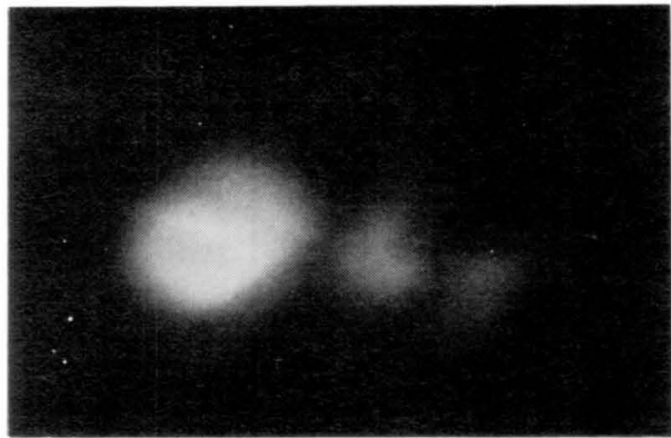

5,1309 UT

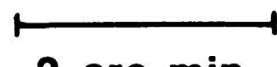

2 arc $\min$.

Fig. 6. McMath 12414 as seen in two coaligned pairs of $\mathrm{H} \alpha$ (top) and soft X-rays (bottom). The July $4 \mathrm{H} \alpha$ is from BBSO and July 5 from Israel. Flux was emerging during this period in the following portion of the region, which consisted of two dipoles bisected by a N-S filament. The EFR was particularly bright in X-rays on July 5. Note how the X-ray loops were aligned along the E-W direction of the H $\alpha$ dark fibrils. The central X-ray emission, especially bright on July 4, arose from an arcade of loops overlying the N-S filament. The right-hand emission coincided with an arcade connecting across the preceding dipole.

data. All of the observed loops connecting to McM 417 terminated in outlying magnetic regions of weak field strength (arrows B, C, D, Figure 4). This is understandable because the sunspot flux and strong field flux is invariably connected to the opposite polarity by stable loops, and only weaker fields on the periphery can be reconnected to external regions. The following examples illustrate the kinds of interconnections we observed.

\subsubsection{Loops Connecting McMath 417 and 414}

McM 417 and 414 were connected by faint transequatorial loops until July 2, $21: 40 \mathrm{UT}$, when a bright connection between $f$ polarity in the following dipole of 
414 and outlying $f$ plage of 417 appeared. This connection brightened considerably by $11: 10$ UT on July 3 and then faded. No flares occurred in this area, but a filament appeared beneath the loop. We surmize that the loop brightening was caused by the trapping of material in the filament neutral sheet.

Another prominent connection took place between the $p$ dipole of AR 414 and an ephemeral region between 414 and 417 . This region appeared as an X-ray bright knot on July 2 which faded on July 3 ; the connecting loop remained, however. No flares occurred in the $\mathrm{H} \alpha$ ephemeral region. This loop brightening was probably linked with the concentrated changing field of the ephemeral region at one footpoint.

In the following days more connections between AR 414 and 417 appeared including the connection of the $p$ dipole of 414 with the $p$ field of 417 . The interconnections between the $f$ plage of McM 414 and $417 f$ went to the same area of outlying plage in 417 (arrow C, Figure 5). This was the nearest place in $417 f$ that a loop from $414 f$ could connect to. On the high resolution $\mathrm{H} \alpha$ pictures the fibrils attached to the $417 f$ plage all pointed toward 414 . Clearly a whole hierarchy of loops connected these regions, but only some were filled with hot emitting material and therefore visible in X-rays at any particular time.

\subsubsection{The Loop Connecting McMath 427 with 417}

The new EFR, McM 427, rapidly formed a loop connection to 417. In $\mathrm{H} \alpha$ from 16:30 to 16:55 UT a discontinuous change in the region occurred; arch filaments appeared and grew rapidly. By 21:09 UT it was a true EFR with small pores, and the $\mathrm{X}$-ray images showed a weakly emitting loop connection to 417 . By the beginning of Tel Aviv observations at $07: 04$ UT on July 6, McM 427 was the brightest region on the Sun in $\mathrm{H} \alpha$, with a small $p$ spot and $\mathrm{H} \alpha$ fibrils extending in all directions.

Continuing surge and subflare activity in McM 427 was probably the source for material in the bright interconnecting X-ray arch that we observed first at 21:09 UT on July 5, only five hours after the birth of 427. By 13:52 UT on July 6 the arch was very bright in $X$-rays (Figure 5 b), following numerous flares in 427 which began about 06:00 UT and continued for the next two days. There was no activity at the other footpoint in 417 .

\subsubsection{A Case for Reconnection}

Howard and Švestka and others have discussed whether the rapid appearance of the loop(s) interconnecting McM regions 417 and 427 was due to reconnection of existing field lines with the EFR, or brightening in situ of pre-existing field lines. Answering this question is difficult, especially with our low X-ray time resolution; the problem of finding unambiguous observational proof of reconnection may in fact be insoluble (Nolte et al., 1977). However, we find the data to be suggestive of reconnection in this case. First, a long, faint, long-lived loop connected the outer edge of the $f$ plage in 417 with a piece of isolated, old $p$ polarity in the quiet Sun. After the emergence of 427 and the interconnecting loop, which intersected the old loop along the line of sight, the original loop was no longer visible, even on the 
longest exposed X-ray images. Second, the feet of the 'old' and 'new' (427-417) loops in 417 were cospatial. Third, the 'new' interconnecting loop bridged the closest opposite polarity field in each region; i.e., a dipole connection was formed across the line of least resistance. This distance was on the order of only two supergranular cells. Finally, initially the 'new' loop's geometry was very non-potential. In its earliest phase, the loop appeared to be long and flattened, i.e., the height-to-length ratio was very small, with an inflection at the McM 427 end. Later as if faded, the loop assumed a more potential configuration. This observation suggests that the loop was relaxing from an initial, highly stressed configuration, as might be expected following rapid reconnection. Figure 1 in Nolte et al. illustrates these observations well.

\subsection{COMPARISONS IN THE CHROMOSPHERE, TRANSITION ZONE AND CORONA}

We compared our data with EUV spectroheliograms obtained on July 4, 6, and 7 (Table I) in the prime polychromatic mode of the Skylab S-055 instrument (Reeves et al., 1977). Emission lines characteristic of the temperature range of the transition zone and low corona were obtained in $5 \times 5$ arc min rasters scanned in C III $977 \AA$ $\left(8 \times 10^{4} \mathrm{~K}\right)$, O IV $554 \AA\left(2 \times 10^{5} \mathrm{~K}\right)$, O vi $1032 \AA\left(3 \times 10^{5} \mathrm{~K}\right)$, and $\mathrm{Mg} \times 625 \AA$ $\left(\leqslant 1.5 \times 10^{6} \mathrm{~K}\right)$. The electron temperatures are characteristic of the maximum concentration for the given ion.

General results observed on every day were: (1) In the cooler lines, but especially the $\mathrm{O}$ lines, the bright, general emission mimicked that of the $\mathrm{H} \alpha$ plage. The brightest spots were also the brightest in $\mathrm{H} \alpha$. Therefore, in the AR transition zone, most of the radiated energy is associated with the low-lying vertical field and little with horizontal field structures. (2) The sunspot and a 'plume', probably the cool legs of far-reaching loops, were in emission in the $\mathrm{O}$ lines. The spot occasionally had bright $\mathrm{C}$ III patches over it. Large-scale loops fanned out from the umbra in $\mathrm{Mg} \mathrm{x}$ and were especially evident to the west of the spot. These loops were apparently cooler than coronal temperatures; they were not observed in X-rays. (3) The coronal (hot) loops were generally uncorrelated with specific EUV features at their bases. When present, bright EUV footpoints were usually cospatial with $\mathrm{H} \alpha$ brightenings, and were at one foot only.

Other specific observations were: (1) On July 4 and 6 bright patches in C III and the $\mathrm{O}$ lines corresponded to areas of high field gradient where there was intrusion of flux of opposite polarity or emerging flux. (2) On July 7 the dominant emission in C III came from an area in the penumbra to the west of the spot. The $\mathrm{H} \alpha$ movie showed that this area was the scene of surging and flaring during the day. It is known that surges can be bright in C III (Kirshner and Noyes, 1971).

\section{Summary and Conclusions}

We studied a simple, evolving active region over sufficient wavelengths to permit mapping of the coronal structures down to the chromosphere/transition region and 
unambiguously to establish the polarities of footpoints in the photospheric field. We classified the loop structures of this particular active region into five categories:

(1) The majority of the brighter loops were internal to the AR and connected regions of bright $f$ plage to either the spot penumbra or to $p$ satellite spots. No non-flaring X-ray loops ended in the umbra.

(2) Persistent, short, bright loops had one or both ends imbedded in regions of emergent flux, strong fields and/or high field gradients. All had one footpoint in the penumbra.

(3) Stable, but sometimes highly sheared arcades arched over filaments and neutral lines. The longest-lived bright loop in the AR was associated with a stable filament.

(4) EFRs were always associated with compact X-ray arcades. Bright loops were aligned with dark $\mathrm{H} \alpha$ fibrils and along the magnetic field.

(5) Loops interconnecting ARs ended in outlying plage of weak field strength in McM 417. $\mathrm{H} \alpha$ fibrils in these regions pointed in the direction of the connecting loops.

We expect the corona over an AR to be filled with magnetic loops. What makes only some of these loops bright in X-rays at a given time? What can we say about the footpoints of the bright loops? We conclude that coronal loops in ARs brighten in response to activity at their bases (filament arcades may be an exception). The brighter X-ray loops always had one or both feet in areas of especially strong and/or changing magnetic fields, such as EFRs, satellite spots, pores or the penumbra, places where lines of force converge. This suggests that the heated material must be contained by strong fields and a favorable geometry in order to produce a brightened loop. X-ray AR loops tend to emit over a relatively narrow temperature range; increases in brightness are due mainly to increases in the emission measure of the loops (Pye et al., 1978; Little and Krieger, 1977). This implies for a constant loop volume an increase of the mass flux into the loop. We suggest that convection of material into the loop is occurring as a result of changing, strong fields and chromospheric heating at the foot of the loop. Such models have been developed for flaring loops (e.g., Moore et al., 1980).

We confirmed previous X-ray observations that non-flaring coronal (hot) loops are never in emission over large stable sun-spots, despite the fact that many magnetic loops must end there. This is the case even if the other foot lies in an active magnetic area. Curiously, dark $\mathrm{H} \alpha$ loops also never end in spots, except in the case of post-flare loops. Presumably the cool sunspot prevents convection of material into the corona by acting like a refrigerator. Pressure-driven siphon flows from active plage areas to the sunspot heat sink might help to evacuate and thereby 'cool' such coronal loops. Conversely we find that small satellite spots and pores can be at the base of quite intense loops; the brightest loops often terminate in the penumbra. In most cases these loops are associated with bright spots in the penumbra. The nature of these spots is unknown, but they may be imbedded satellite sunspots which are known to be connected with flares (Rust, 1972). 
It is of interest to compare these observations with radio data. It is well known that the slowly varying component of the radio Sun peaks at centimeter wavelengths over active regions. With only moderate spatial resolution (i.e., $\geq 6$ arc sec), many observers have concluded that this microwave emission peaks directly over spot umbrae (see Schmahl, 1980, for a review). The popular model of gyroresonance absorption as the source for the microwave emission predicts a high temperature, low density plasma in a high magnetic field over a sunspot. The observation of large columns of cool EUV material and the absence of X-radiation over umbrae appears to cause a problem with the model. However, using VLA data, Kundu and Velusamy (1980) and Kundu et al. (1980) recently have observed ARs where the brightest $6 \mathrm{~cm}$ features occur over the penumbra, and are probably associated with hot loops. Kundu et al. conclude that this displacement is consistent with both gyroresonance absorption in hot loops and our observations of bright penumbral loops.

The largest X-ray loops in the AR form filament arcades. These arcades form a unique geometrical class of loops. However, single arcade loops also can brighten in response to strong field activity at their feet, such as the moving magnetic feature on July 5 and 6 (Section 3.3). This observation tends to support the model developed by Davis and Krieger (1980) in which at least some AR filaments grow by the condensation of material from unstable dense coronal arches which precede the filament formation by hours. The mechanism which provides material to the arches may be convection from magnetically active areas at the feet of the arches. We note that the arcades over quiescent filament channels outside of ARs are always faint and diffuse on the disk (McIntosh et al., 1976). This may be because the fields at their bases are weaker, more diffuse and relatively inactive compared with those in ARs.

Our results show that sheared arcade loops, possibly with twists in them, can exist in ARs in a long-term stable configuration. Thus a sheared configuration is not a sufficient condition for filament disruption. In fact certain empirical models (e.g., Rust, 1976) predict a high degree of shear in low-lying, stable filament structures. The inflection in the loop overlying filament No. 1 implies a twist which shows that the field is not poloidal. This configuration may enhance the ability of the arcade loop to confine material and therefore to brighten.

Emerging flux was always associated with compact X-ray arcades, which typically expanded with the $\mathrm{H} \alpha$ EFR area. When resolved, the $\mathrm{X}$-ray loops were aligned with the arch filaments, i.e., a simple dipole field existed with little vertical shear. We believe that EFR development resembles that of a loop prominence system. Coronal loops form as flux is brought to the surface and the loops are heated, possibly by reconnection; the loops radiate energy and cool to form $\mathrm{H} \alpha$ arches. Since the field is concentrated and arcades coexist at different heights, the plage appears as a single bright blob with only the highest loops discernable.

$\mathrm{X}$-ray loop interconnections appeared within a few hours of the birth of an isolated EFR. The interconnections terminated in AR 417 in well-defined areas of the outlying plage, where $\mathrm{H} \alpha$ fibrils pointed in the direction of the interconnection (Figure 5a, Arrow B). In every case of an EFR we studied, outward pointing fibrils 
appeared on the edge of the $p$ spot in the first few days. We suggest that these were the feet of the loops connecting to distant points.

There seemed to be different sources for interconnection loop brightenings. The AR 427-417 connection was undoubtedly enhanced by emerging flux and flaring in AR 427 and possibly by Joule heating following reconnection. Other brightenings appeared to be influenced by an ephemeral region, by general activity and possibly by the trapping of material in loops over an equatorial filament.

In conclusion, we present the following interpretation for why certain coronal $A R$ loops are bright at any given time. Lines of force fill most of the corona over an AR at all times. As a result of the emergence or convergence of strong magnetic fields in the photosphere and chromosphere, reconnection or other processes occur which heat the chromosphere. If this heating occurs at the base of a suitably positioned flux loop, the heated material will convect up the loop, increasing its X-ray emissivity. The loop will shine so long as the heating continues and will cool once the activity at its base stops. Since radiative cooling of long loops takes many hours, and magnetic activity in a young $\mathrm{AR}$ is persistent, at any one time loops will be in various stages of heating and cooling with a resultant range in brightness.

We found evidence that filament arcade loops and interconnecting loops are heated at least occasionally by this same process. However, these loops are large and will cool more slowly than the short internal AR loops. In addition, the interconnecting loops are visible against the quiet coronal background. Therefore, these two classes of loops will appear to be more stable (longer-lived) than the others.

\section{Acknowledgements}

This study was initiated during the authors' participation in the Skylab Solar Workshop on Active Regions. The Workshops are sponsored by NASA and NSF and managed by the High Altitude Observatory. We thank the members of the AS\&E Photographic Laboratory for their assistance with the imagery used in this study. We are grateful to R. Levine of the Harvard-Smithsonian Center for Astrophysics for providing S-055 data. DFW benefited by helpful discussions with A. Krieger and was supported by NASA Contract NAS8-27758. HZ was supported by NASA under NGL 05-002-034, by NSF under ATM 76-21132 and by the Air Force under F-19628-77-C-0106.

\section{References}

Chase, R. C., Krieger, A. S., Švestka, Z., and Vaiana, G. S.: 1976, Space Research XVI, 917.

Davis, J. M. and Krieger, A. S.: 1980, Solar Phys. (submitted).

Howard, R. and Švestka, Z.: 1977, Solar Phys. 54, 65.

Kahler, S. W., Webb, D. F., and Moore, R. L.: 1981, Solar Phys. (in press).

Kirshner, R. P. and Noyes, R. W.: 1971, Solar Phys. 20, 428.

Krieger, A. S., Vaiana, G. S., and Van Speybroeck, L. P.: 1971, in R. Howard (ed.), 'Solar Magnetic Fields', IAU Symp. 43, 397.

Krieger, A., Paolini, F., Vaiana, G. S., and Webb, D.: 1972, Solar Phys. 22, 150. 
Krieger, A. S., de Feiter, L. D., and Vaiana, G. S.: 1976, Solar Phys. 47, 117.

Kundu, M. R. and Velusamy, T.: 1980, Astrophys. J. Letters 240, L63.

Kundu, M. R., Schmahl, E. J., and Rao, A. P.: 1980, Astron. Astrophys. (in press).

Little, S. J. and Krieger, A. S.: 1977, Bull. Am. Astron. Soc. 9, 341.

McIntosh, P. S., Krieger, A. S., Nolte, J. T., and Vaiana, G.: 1976, Solar Phys. $49,57$.

Moore, R. L., McKenzie, D. L., Švestka, Z., Widing, K. G., Antiochus, S. K., Dere, K. P., Dodson-Prince, H. W., Hiei, E., Krall, K. R., Krieger, A. S., Mason, H. E., Petrasso, R. D., Pneuman, G. W., Silk, J. K., Vorpahl, J. A., and Withbroe, G. L.: 1980, in P. A. Sturrock (ed.), Solar Flares, Colorado Associated University Press, Boulder, Colo.

Neupert, W. M., Nakagawa, Y., and Rust, D. M.: 1975, Solar Phys. 43, 359.

Nolte, J. T., Gerassimenko, M., Krieger, A. S., Petrasso, R. D., Švestka, Z., and Wentzel, D. G.: 1977, Solar Phys. 55, 401.

Pallavicini, R., Vaiana, G. S., Tofani, G., and Felli, M.: 1979, Astrophys. J. 229, 375.

Pye, J. P., Evans, K. D., Hutcheon, R. J., Gerassimenko, M., Davis, J. M., Krieger, A. S., and Vesecky, J. F.: 1978, Astron. Astrophys. 65, 123.

Reeves, E. M., Huber, M. C. E., and Timothy, J. G.: 1977, Appl. Opt. 16, 837.

Roy, J. R. and Michalitsanos, A. G.: 1974, Solar Phys. 35, 47.

Rust, D. M.: 1972, Solar Phys. 25, 141.

Rust, D. M.: 1976, Solar Phys. 47, 21.

Sheeley, N. R., Bohlin, J. D., Brueckner, G. E., Purcell, J. D., Scherrer, V., and Tousey, R.: 1975, Solar Phys. 40, 103.

Schmahl, E.: 1980, in M. Kundu and T. Gergeley (eds.), 'Radio Physics of the Sun', IAU Symp. 86, 71.

Smith, J. B., Speich, D. M., Tandberg-Hanssen, E., Wilson, R. M., de Loach, A. C., Hoover, R. B., McGuire, J. P., Henze, W., and Wu, S. T.: 1976, Space Research XVI, 903.

Tang, F.: 1978, Solar Phys. 70, 119.

Tousey, R., Bartoe, J., Bohlin, J. D., Brueckner, G. E., Purcell, J. D., Scherrer, V. E., Sheeley, N. R., Schumacher, R. J., and Van Hoosier, M. E.: 1973, Solar Phys. 33, 265.

Van Speybroeck, L. P., Krieger, A. S., and Vaiana, G. S.: 1970, Nature 227, 818.

Vaiana, G. S., Van Speybroeck, L., Zombeck, M. V., Krieger, A. S., Silk, J. K., and Timothy, A.: 1977, Space Sci. Instrum. 3, 19.

Weart, S. R.: 1970, Astrophys. J. 162, 987.

Zirin, H.: 1974, in R. G. Athay (ed.), 'Chromospheric Fine Structure', IAU Symp. 56, 161. 